\title{
Increased production of IL-7 uncouples bone formation from bone resorption during estrogen deficiency
}

\author{
M. Neale Weitzmann, Cristiana Roggia, Gianluca Toraldo, Louise Weitzmann, \\ and Roberto Pacifici
}

Division of Bone and Mineral Diseases, Washington University School of Medicine and Barnes-Jewish Hospital, St. Louis, Missouri, USA

\begin{abstract}
Postmenopausal bone loss stems from the inability of osteoblastic activity to match the increase in osteoclastic bone resorption induced by estrogen deficiency. However, the mechanism that uncouples osteoblast from osteoclast activities remains unexplained. We show that ovariectomy enhances the production of the osteoclastogenic cytokine IL-7, and that its neutralization in vivo prevents ovariectomy-induced bone loss. Surprisingly, serum osteocalcin levels, a biochemical marker of bone formation, suggested that the bone-sparing effects of IL-7 neutralization were due not only to inhibition of bone resorption, but also to stimulation of bone formation. Consistent with these data, addition of IL-7 to neonatal calvarial organ cultures blocked new bone formation, and injection of IL-7 into mice in vivo inhibited bone formation as measured by calcein incorporation into long bones. The antianabolic effects of IL-7 were consistent with an observed downregulation of the osteoblast-specific transcription factor core-binding factor $\alpha 1 /$ Runx 2 . Thus, because it targets both the osteoclast and the osteoblast pathways, IL-7 is central to the altered bone turnover characteristic of estrogen deficiency.
\end{abstract}

J. Clin. Invest. 110:1643-1650 (2002). doi:10.1172/JCI200215687.

\section{Introduction}

Postmenopausal bone loss is a consequence of defective bone remodeling stemming from estrogen (E2) deficiency. The withdrawal of sex steroid leads to bone loss because bone formation, albeit enhanced, is unable to keep pace with an even more abundant stimulation of osteoclastic bone resorption (1), a phenomenon known as uncoupling. The mechanism driving uncoupling is central to the pathogenesis of postmenopausal osteoporosis but remains poorly understood.

Several cell lineages and multiple cytokines have been implicated in the mechanism by which E2 deficiency upregulates osteoclast (OC) formation. IL-7 is an osteoclastogenic cytokine (2) best recognized for its ability to stimulate $B$ and $T$ cells (3). In the bone marrow, stromal cells (4) and osteoblasts (OBs) (5) are the principal source of IL-7. IL-7 promotes osteoclastogenesis by upregulating key $\mathrm{T}$ cell-derived osteoclastogenic cytokines, including receptor activator of NF- $\mathrm{KB}$

Received for publication April 15, 2002, and accepted in revised form September 24, 2002.

Address correspondence to: M. Neale Weitzmann, Division of Bone and Mineral Diseases, Barnes-Jewish Hospital, MS 90-32-656, 216 S. Kingshighway Boulevard, St. Louis, Missouri 63110, USA. Phone: (314) 454-8829;

Fax: (314) 454-5047; E-mail: nweitzmann@usa.net.

Conflict of interest: The authors have declared that no conflict of interest exists.

Nonstandard abbreviations used: estrogen (E2); osteoclast (OC); osteoblast (OB); receptor activator of NF- $\mathrm{KB}$ ligand (RANKL); ovariectomy(ized) (ovx); osteocalcin (OCN); corebinding factor $\alpha 1$ (Cbfa1); osteoprotegerin (OPG); bone mineral density (BMD); dual energy $x$-ray absorptiometry (DEXA); deoxypyridinoline (DPD). ligand (RANKL) (5). IL-7 also increases the number of early precursors of the $\mathrm{B}$ cell lineage (B220 cells) in the bone marrow (2), a population that has been suggested to have the capacity to differentiate into OCs (6). Thus IL-7 may also induce bone loss by increasing the pool of OC precursors.

The mechanism by which bone formation is uncoupled from bone resorption following ovariectomy (ovx) is incompletely characterized. OBs are cells of mesenchymal origin that differentiate into cells expressing characteristic markers of the OB phenotype, including alkaline phosphatase, bone sialoprotein, osteopontin, and type I collagen (7). These proteins are involved in and necessary for matrix production and deposition, a principal function of the OB. Mineralization of deposited matrix is an important functional characteristic of the $\mathrm{OB}$ and is strongly associated with the expression of osteocalcin $(\mathrm{OCN})$, a negative regulator of and specific marker of OB activity (7). The OB-specific transcription factor core-binding factor $\alpha 1 /$ Runx2 (Cbfa1/Runx2) has been found to play a prominent role in the transactivation of the OCN promoter as well as in a number of other important OB genes, including bone sialoprotein, osteopontin, and type I collagen (8-10), and has been reported to stimulate the OB production of the RANKL inhibitor osteoprotegerin (OPG) (11).

In this study we examined the role of IL-7 in the bone loss associated with E2 deficiency and investigated the mechanisms by which IL-7 uncouples bone formation from bone resorption. We propose a novel model, based on our data, whereby upregulation of IL-7, a consequence of E2 deficiency, leads to induction of osteoclastogenesis, while simultaneously repressing $\mathrm{OB}$ 
differentiation and activity, thus limiting the magnitude of the compensatory increase in bone formation necessary to restore homeostasis.

\section{Methods}

All animal procedures were approved by the Animal Studies Committee of Washington University. All reagents were purchased from Sigma Chemical Co. (St. Louis, Missouri, USA), unless otherwise indicated.

IL-7 neutralization in vivo. To block IL-7 in vivo, C57BL/ 6 mice (Taconic, Germantown, New York, USA) were ovx as previously described (12) and treated with an anti-IL-7 antibody (M25) previously shown to neutralize IL-7 in vivo $(13,14)$ (kindly provided by Stewart D. Lyman, Immunex Corp., Seattle, Washington, USA). Control ovx mice received nothing or an irrelevant isotype-matched (IgG2b) antibody. Mice were injected intraperitoneally with $1 \mathrm{mg}$ of protein A-purified M25 ascites three times per week for 4 weeks. Success of the ovx was confirmed at sacrifice by atrophy of the uterus. Ovx also results in a 50\% increase in spleen mass. Successful M25 neutralization was assessed by prevention of ovx-induced increase in spleen mass.

Bone mineral density. In vivo bone mineral density (BMD) measurements of the femur heads were made in anesthetized mice at base line ( 0 time) and at 2 and 4 weeks after ovx by dual energy $x$-ray absorptiometry (DEXA) using a Hologic 2000 bone densitometer with dedicated software for analysis of small animals (Hologic Inc., Bedford, Massachusetts, USA), as previously described (15).

OCN assays. OCN, a sensitive biochemical marker of bone formation, was measured in the serum of all groups of mice 4 weeks after surgery using the RIA of Biomedical Technologies Inc. (Stoughton, Massachusetts, USA).

Deoxypyridinoline cross-link assays. Deoxypyridinoline (DPD) cross-link was measured in the urine of all groups of mice 4 weeks after surgery by Pyrilinks-D assay ELISA (Metra Biosystems Inc., Mountain View, California, USA). Results were corrected for urinary concentration by standardizing with creatinine as described $(12,15)$.

IL-7 ELISA. A murine IL-7 ELISA was generated using M25 mAb (Immunex Corp.) as capture antibody (5 $\mu \mathrm{g} / \mathrm{ml}$ in PBS; $100 \mu \mathrm{l} /$ well), anti-IL-7-biotin (R\&D Systems Inc., Minneapolis, Minnesota, USA) as detection antibody $(5 \mu \mathrm{g} / \mathrm{ml}$ in $100 \mu \mathrm{l} /$ well final), and $1 \mu \mathrm{g} / \mathrm{ml}$ avidin-peroxidase (Sigma Chemical Co.) as conjugate. A solution consisting of $0.015 \%$ 2,2'-azino-bis(3-ethylbenzthiazoline- 6 sulphonic acid containing $1 \mu \mathrm{l} / \mathrm{ml}$ $\mathrm{H}_{2} \mathrm{O}_{2}(30 \% \mathrm{vol} / \mathrm{vol})$ was used for color development. Standards (recombinant murine IL-7; R\&D Systems Inc.) and experimental samples were added in a final volume of $100 \mu \mathrm{l}$, diluted in assay buffer (4\% BSA in PBS). The ELISA was read at $\mathrm{OD}_{410}$ on an automated SPECTRAmax ${ }^{250}$ ELISA reader (Molecular Devices Corp., Sunnyvale, California, USA). The sensitivity of the ELISA is typically $\leq 50 \mathrm{pg} / \mathrm{ml}$. No cross-reactivity was observed by stem cell factor, M-CSF, GM-CSF, IL-1 $\alpha$, IL-2, IL-6, IL-12, RANKL, OPG, IFN- $\gamma$, TNF- $\alpha$, and
TGF- $\beta$, each assayed at $5 \mathrm{ng} / \mathrm{ml}$. Intra-assay precision coefficient of variation (\%) of five identical samples was $\leq 7 \%$. Interassay precision of identical samples, measured in three independent assays, was $\leq 10 \%$. To further validate our ELISA, we measured the concentration of IL-7 in the serum of a heterozygous and a homozygous IL-7 knockout mouse. While an average concentration of $750 \mathrm{pg}$ of IL-7 was detected in the serum of the heterozygous mouse, no IL-7 was detected in the homozygous knockout mouse.

Immediately following isolation without further in vitro culture, whole bone marrow from mouse femurs was isolated by centrifugation and IL-7 was measured in the cell-free bone marrow supernatant, thus providing an in vivo measure of the state of IL-7 production in the bone marrow.

Semiquantitative RT-PCR. Total RNA from ROS $17 / 2.8$ cells or whole bone marrow was extracted using Trizol reagent (GIBCO BRL; Life Technologies Inc., Gaithersburg, Maryland, USA) according to the manufacturer's instructions. Semiquantitative RT-PCR was conducted using Ready-To-Go RT-PCR beads (Amersham Pharmacia Biotech., Piscataway, New Jersey, USA) according to the manufacturer's instructions. The number of cycles necessary to amplify cDNA but remain below saturation was determined for each primer set and cell type. The primers used were mouse IL-7 forward 5'-TATTCTAGAATGTTCCATGTTTCTTTTAGA- $3^{\prime}$ and reverse $5^{\prime}$-CTTTTTCTGTTCCTTTA- $3^{\prime}$ and rat OPG forward $5^{\prime}$-ATGAACAAGTGGCTGTGCTG- $3^{\prime}$ and reverse $5^{\prime}$-GGCCTGATGTACGCACTCAA- $3^{\prime}$. Commercial murine GAPDH primers (CLONTECH Laboratories Inc., Palo Alta, California, USA) were used as an internal standard to ensure that equal RNA concentrations were used in each sample. Genomic contamination was assessed by heat inactivation of the RT prior to RT-PCR. Bands were scanned on an Epson flatbed scanner (Epson America Inc., Long Beach, California, USA), and OD was quantitated using NIH Image version $1.62 \mathrm{f}$ for Macintosh (Research Services Branch of the National Institute of Mental Health, NIH).

OPG Western blot. Murine calvarial OBs were isolated from fetal mouse calvaria by sequential collagenase digestion and seeded in BGJ media supplemented with $0.01 \%$ BSA as previously described (16). After 24 hours' stimulation with IL-7 $(20 \mathrm{ng} / \mathrm{ml})$, cytosolic protein was extracted as described (17), $50 \mu \mathrm{g}$ of protein (determined by Bradford assay) from unstimulated and IL-7-treated groups was electrophoresed on 4-20\% gradient SDS-PAGE gels, and Western blot was performed using mouse anti-OPG (clone H249) and horseradish peroxidase-conjugated rabbit anti-mouse IgG secondary antibody (Santa Cruz Biotechnology Inc., Santa Cruz, California, USA). OPG was visualized using SuperSignal West Pico chemiluminescence substrate (Pierce Chemical Co., Rockford, Illinois, USA). Bands were scanned on an Epson flatbed scanner, and OD was quantitated using NIH Image version $1.62 \mathrm{f}$ 
for Macintosh. Even protein loading was confirmed by Coomassie blue staining of the gels following transfer onto nitrocellulose membranes.

Cbfa1/Runx2 promoter cloning. The distal OB-specific mouse Cbfa1/Runx 2 promoter (-620 bp) was cloned by PCR from genomic mouse DNA using primers based on the published sequence for Cbfa1/Runx2 (18). The forward primer was $5^{\prime}$-GCATGCTAGCGCAGCACTGTTGCTCAGAACGCC-3', and the reverse primer was $5^{\prime}$ CGATCTCGAGTGGCTGGTAGTGACCTGCAGAGATTA-3'. Cbfa1/Runx2 was digested with NheI and XhoI and cloned into pGL3-basic luciferase vector. Positive clones were confirmed by DNA sequencing from both ends. Transfection and dual luciferase assays. ROS 17/2.8 cells were transfected using SuperFect reagent (QIAGEN Inc., Valencia, California, USA) with $1.5 \mu \mathrm{g}$ of one of the following: DNA (pGL3-basic empty vector control); pGL3-basic vector containing the distal OB-specific Cbfa1/Runx2 promoter (pGL3-Cbfa1/Runx2), cloned as described above; p6OSE2-luc, a construct containing six copies of the OSE 2 motif coupled to a minimal OCN promoter; or p6OSE2mut-luc, with six mutated OSE2 motifs incapable of binding Cbfa1/Runx2. p6OSE2-luc and p6OSE2mut-luc were kindly provided by Gerard Karsenty (Baylor College of Medicine, Houston, Texas, USA) and have been previously described (19). Each reaction also contained $0.2 \mu \mathrm{g}$ of pRL-SV40 internal control, a construct expressing Renilla luciferase (Promega Corp., Madison, Wisconsin, USA). Following transfection, cells were stimulated with vehicle (PBS plus $0.1 \% \mathrm{BSA}$ ) or $10 \mathrm{ng} / \mathrm{ml}$ recombinant murine IL-7, and luciferase activity was measured after 24 hours on a Lumat LB 9507 luminometer (Berthold Technologies $\mathrm{GmbH}, \mathrm{Bad}$ Wildbad, Germany). Results were adjusted for transfection efficiency using the cotransfected Renilla construct as internal control. Data are expressed as normalized luciferase activity.

Calvarial organ culture. Calvarial organ cultures were conducted as previously described (20). Calvaria were cultured for 2 weeks in the presence or absence of 50 $\mathrm{ng} / \mathrm{ml}$ murine IL-7 (R\&D Systems Inc.) and/or $50 \mathrm{ng} / \mathrm{ml}$ bone morphogenetic protein-2 (BMP2) (Genetics Institute Inc., Cambridge, Massachusetts, USA). Calvaria were fixed in $10 \%$ formalin for 2 hours, decalcified in EDTA overnight, and embedded in paraffin wax, and sections were cut along the laterally bisected region such that comparisons were made at equivalent positions in each sample. Sections were stained with hematoxylin and eosin and photographed at $\times 100$ magnification under phase-contrast microscopy.

In vivo IL-7 administration and calcein labeling. Six-weekold C57BL/6 mice from Taconic (four mice per group) were injected intraperitoneally daily for 30 days with 10 $\mu \mathrm{g} / \mathrm{kg}$ body weight of recombinant human IL-7 (generously provided by Adrian Minty, Sanofi-Synthelabo SA, Paris, France) or with vehicle (PBS), as previously described (2) but with modifications; they also received a double pulse of calcein $\left(20 \mathrm{mg} / \mathrm{kg}\right.$ in $\left.2 \% \mathrm{NaHCO}_{3}\right) 10$ and 3 days prior to sacrifice. Tibiae were isolated, fixed in $70 \%$ ethanol, and embedded in polymethyl methacrylate resin for histological sectioning. Longitudinal sections were prepared and photographed at comparable anatomic positions at mid-diaphysis $(1 \mathrm{~mm}$ in length) using a Zeiss Axiovert S100 fluorescence microscope (Carl Zeiss Inc., Thornwood, New York, USA) at $\times 100$ magnification. Digital color images were converted to black and white, and the intensity of calcein fluorescence over the entire area of each of the four independent samples for each group was quantitated and averaged using NIH Image version $1.62 \mathrm{f}$ software.

Statistics. Cross-sectional BMD data were analyzed by ANOVA and Fisher protected least significant difference tests. Simple comparisons were made using twotailed $t$ test. $P \leq 0.05$ was considered significant.

\section{Results}

E2 deficiency upregulates IL-7 levels in the bone marrow. IL-7 has been reported to mimic many of the actions of E2 deficiency, including upregulation of $\mathrm{B} 220^{+}$cells, induction of bone loss (2), and stimulation of osteoclastogenic cytokines from $\mathrm{T}$ cells (5). We thus investigated

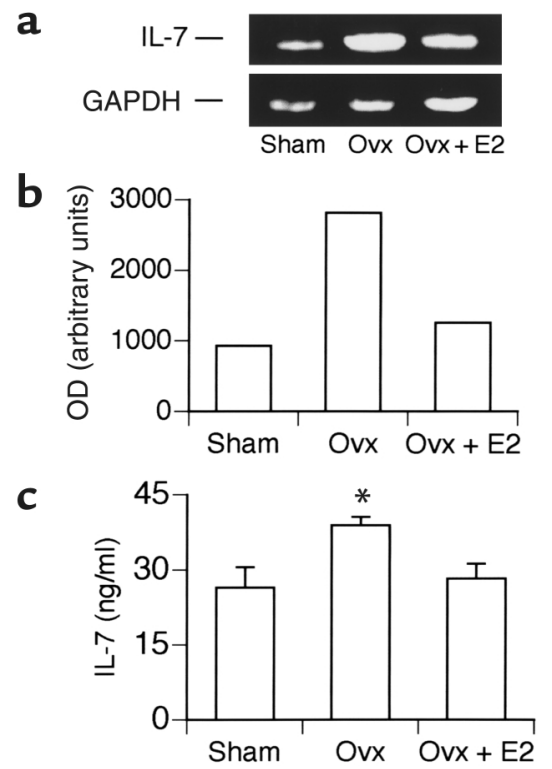

\section{Figure 1}

E2 deficiency upregulates IL-7 mRNA and protein concentrations in murine bone marrow cells in vivo. (a) Levels of IL-7 mRNA were analyzed by semiquantitative RT-PCR using whole murine bone marrow isolated from E2-deficient ovx mice and E2-replete mice (sham-operated, and ovx receiving E2 replacement) 2 weeks after surgery $(n=4$ mice per group). RNA concentrations were internally controlled using the housekeeping gene GAPDH. IL-7 was evaluated at 30 PCR cycles and GAPDH at 25. IL-7 was confirmed to be below saturation up to 35 cycles and GAPDH up to 30 cycles (data not shown). Data are representative of two independent experiments. (b) Quantitation of IL-7 mRNA. IL-7 mRNA bands in a were quantitated by densitometry using $\mathrm{NIH}$ Image and normalized for GAPDH concentrations. (c) IL-7 protein concentrations in the marrow compartments of E2-replete and E2-deficient mice were measured by ELISA in cell-free bone marrow serum immediately following isolation of the marrow, as described in Methods. Data represent average \pm SEM of four mice per group. Data points were measured in quadruplicate. ${ }^{*} P \leq 0.05$ (ANOVA). 


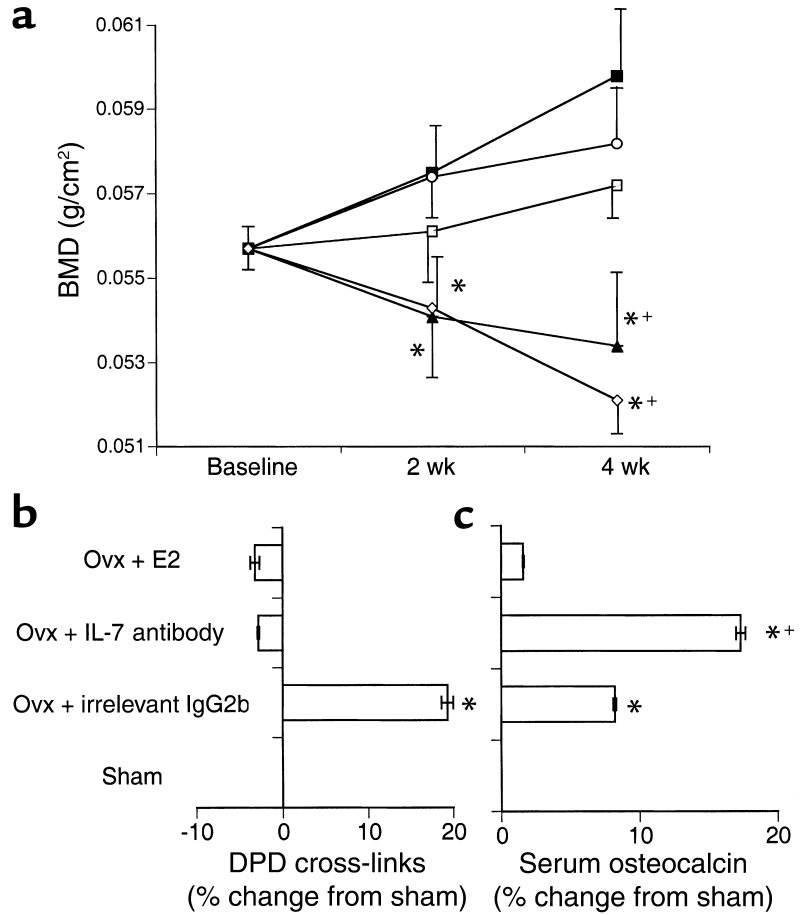

Figure 2

IL-7 neutralization in vivo prevents bone loss following ovx. (a) Mice were sham-operated (filled squares) or ovx-operated (open diamonds), with one additional ovx group receiving E2 replacement (open circles). Another group of ovx mice was injected intraperitoneally with $1 \mathrm{mg}$ of anti-IL-7 neutralizing antibody three times per week (open squares), and one group of ovx mice received irrelevant isotype-matched (IgG2b) antibody (filled triangles). BMD $\left(\mathrm{mg} / \mathrm{cm}^{2}\right)$ was measured by DEXA at base line $(0)$ and at 2 and 4 weeks after ovx. Data are shown as average \pm SEM. ${ }^{*} P \leq 0.01 \mathrm{com}-$ pared with sham-operated mice and ovx mice receiving E2 replacement; ${ }^{+} P \leq 0.02$ compared with ovx mice injected with anti-IL-7 antibody (ANOVA; $n=6$ mice per group). (b) The urinary concentration of DPD was measured at 4 weeks after surgery by ELISA, and data were corrected for urinary excretion using creatinine. Data represent average percent change from sham-operated \pm SEM. Samples were measured in triplicate. ${ }^{*} P \leq 0.05$ compared with sham-operated mice (ANOVA; $n=6$ mice per group). (c) Serum $\mathrm{OCN}$ was measured at sacrifice by RIA. Data represent average percent change from sham-operated \pm SEM. Samples were measured in triplicate. ${ }^{*} P \leq 0.05$ compared with sham-operated mice; ${ }^{+} P \leq 0.05$ compared with ovx-operated mice receiving irrelevant $\operatorname{lgG} 2 \mathrm{~b}$ antibody (ANOVA; $n=6$ mice per group).

whether IL-7 production was increased in the bone marrow of ovx mice compared with E2-replete controls. We examined the levels of IL-7 mRNA present in total bone marrow isolated from E2-deficient ovx mice and E2-replete mice (sham-operated, and ovx receiving E2 replacement) 2 weeks after surgery using semiquantitative RT-PCR (Figure 1a). Quantitation of Figure 1a shows a threefold elevation in IL-7 mRNA in the bone marrow of ovx mice compared with both sham-operated and E2-replete ovx groups (Figure 1b).

As there are no commercial ELISAs for murine IL-7, we developed a specific murine IL-7 sandwich ELISA that allowed us to directly measure IL-7 protein levels in the bone marrow compartments of E2-replete and E2-deficient mice. Bone marrow was isolated from mouse femurs by centrifugation. Immediately following isolation, IL-7 was measured directly, without additional in vitro culture, in the cell-free supernatant (serum) thus obtained. This provided a measure of in vivo IL-7 concentrations in the bone marrow. The data show a $35 \%$ increase in IL-7 protein concentrations in ovx bone marrow (Figure 1c).

Together these data support the contention that IL-7 production is upregulated in the bone marrow during E2 deficiency.

IL-7 neutralization in vivo prevents bone loss following ovx. In order to determine whether upregulated levels of IL-7 in ovx mice contribute to the bone loss induced by E2 deficiency, we used a specific neutralizing antibody (M25) directed against IL-7 $(13,14)$ to block IL-7 in vivo following ovx. BMD was measured by DEXA at base line (0) and at 2 and 4 weeks after ovx. The data demonstrate that while ovx mice and ovx mice receiving an irrelevant isotype-matched (IgG2b) antibody show significant bone loss compared with sham-operated mice and ovx mice receiving E2 replacement $(P \leq 0.01)$, ovx mice treated with anti-IL-7 antibody were completely protected from bone loss at 4 weeks after surgery $(P=$ not significant compared with sham-operated mice; $P \leq 0.02$ vs. irrelevant antibody-treated mice) (Figure 2a). Ovx mice injected with an irrelevant isotype-matched (IgG2b) antibody displayed no significant difference in bone loss compared with untreated ovx mice.

IL-7 neutralization prevents bone loss by downregulating bone resorption and stimulating bone formation. In order to determine the effects of IL-7 neutralization on ovxinduced bone resorption in vivo, we measured the urinary excretion of DPD cross-links, a sensitive biochemical marker of bone resorption whose levels closely correlate with histomorphometric measurements of bone resorption (21). As expected, ovx mice showed a significant increase in DPD following ovx $(P \leq 0.01$; Figure $2 b)$. In contrast, neutralization of IL-7 in vivo completely blocked ovx-induced DPD, suggesting that the elevated levels of IL-7 during E2 deficiency act to induce osteoclastic resorption during ovx.

In order to evaluate any effects of IL-7 neutralization on bone formation, we examined the levels of serum OCN, a specific biochemical marker of bone formation (22), 4 weeks after surgery. Surprisingly, OCN levels were found to be significantly increased $(P \leq 0.01)$ in IL-7 antibody-treated mice compared with sham-operated mice receiving irrelevant isotype-matched antibody (Figure 2c). These data suggest that elevated levels of IL-7 act to repress bone formation during ovx.

Together our data suggest that IL-7 is a key protagonist in the bone loss associated with E2 deficiency, achieving its effect by upregulating osteoclastic bone resorption while simultaneously limiting the magnitude of the rise in bone formation necessary to re-establish bone homeostasis. IL-7 hence uncouples bone formation from bone resorption during E2 deficiency. 


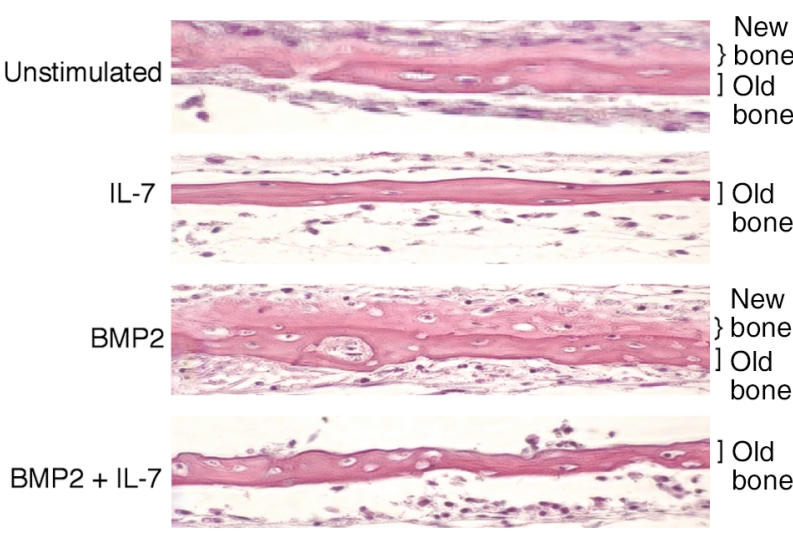

Figure 3

IL-7 inhibits both basal and BMP2-stimulated bone formation in neonatal calvarial organ cultures in vitro. Neonatal calvaria (2 days old) were cultured for 2 weeks in the presence or absence of IL-7 (50 $\mathrm{ng} / \mathrm{ml})$ and/or BMP2 $(50 \mathrm{ng} / \mathrm{ml})$. Calvaria were stained with hematoxylin and eosin and photographed at $\times 100$ magnification. Dark pink bands indicate old bone, while light pink bands represent newly formed bone. Data are representative of two independent experiments.

IL-7 inhibits both basal and BMP2-stimulated bone formation in calvarial organ cultures in vitro. To verify an inhibitory effect of IL-7 on bone formation as indicated by the IL-7 neutralization experiment, we investigated whether IL-7 could blunt new bone growth in neonatal calvaria, using an in vitro organ culture as previously described (20). Calvaria were cultured for 2 weeks in the presence or absence of IL-7 $(50 \mathrm{ng} / \mathrm{ml})$. Additional calvaria were also stimulated with BMP2 $(50 \mathrm{ng} / \mathrm{ml})$, a potent inducer of bone formation (23), in the presence or absence of IL-7. The data show that IL-7 inhibits bone formation compared with that in the untreated control (Figure 3). In addition, IL-7 completely repressed BMP2-stimulated bone formation, thus confirming that IL-7 is a powerful inhibitor of osteoblastic bone formation.

IL-7 inbibits bone formation in mice in vivo. To verify an inhibitory effect of IL-7 in vivo, we investigated whether IL-7 could directly inhibit bone formation when injected into mice. Mice (four per group) were injected daily for 30 days with vehicle (PBS) or $10 \mu \mathrm{g}$ of recombinant human IL-7 per kg body weight, the minimum dose found to elicit a significant bone loss in a preliminary titration. Mice were pulsed 7 and 3 days before sacrifice with calcein, a fluorescent stain of newly synthesized bone in vivo (24), and excised tibiae were processed for histological examination. The data demonstrate that while significant incorporation of calcein occurred in vehicle-treated mice, with clear double labeling observable in the tibiae, mice injected with IL-7 show no visible double labeling and a 93\% reduction in total calcein uptake, as assessed by densitometric quantitation (Figure 4, $a$ and b). These data confirms that IL-7 is a powerful inhibitor of new bone formation in vivo.

IL-7 inhibits the transactivation of a Cbfa1/Runx2-responsive promoter driven by OSE2 elements and blunts Cbfa1/Runx2 promoter activity. As shown above (Figure $2 c)$, our data suggest that in vivo IL-7 blocks OCN production, by blunting the activity of bone-forming cells. The regulation of OCN transcription has been well studied, and the OSE2-binding factor Cbfa1/Runx2 is known to play a crucial role $(25,26)$. We thus investigated whether Cbfa1/Runx2 is downregulated in the osteoblastic cell line ROS 17/2.8 following IL-7 stimulation. We measured bioactive Cbfa1/Runx2 levels by determining the degree of transactivation of a specific OSE2-driven promoter. In this experiment we used a luciferase reporter construct driven by six tandem copies of OSE2 (the binding site for the Cbfa1/Runx2 transcription factor) fused to a minimal OCN promoter (p6OSE2-luc) and an inactive mutant control (p6OSE2mut-luc), which were kindly provided by G. Karsenty and have been previously described (19). The constructs were transfected into ROS 17/2.8 OB-like cells, and luciferase activity was assessed at 24 hours. The data show a high basal rate of transcription induced by Cbfa1/Runx2 in ROS 17/2.8 cells, as has been previously reported $(18,27,28)$. However, upon IL-7 stimulation, OSE2-driven gene activity was suppressed by $50 \%$ (Figure 5a), indicating reduced transactivation of the OSE2 consensus sequence by Cbfa1/Runx2. These data suggest that IL-7 leads to a decrease in Cbfa1/Runx2 activity, a specific marker of OB differentiation.

As Cbfa1/Runx2 activity is regulated by both transcriptional and posttranslational mechanisms, we investigated the state of Cbfa1/Runx2 gene transactivation
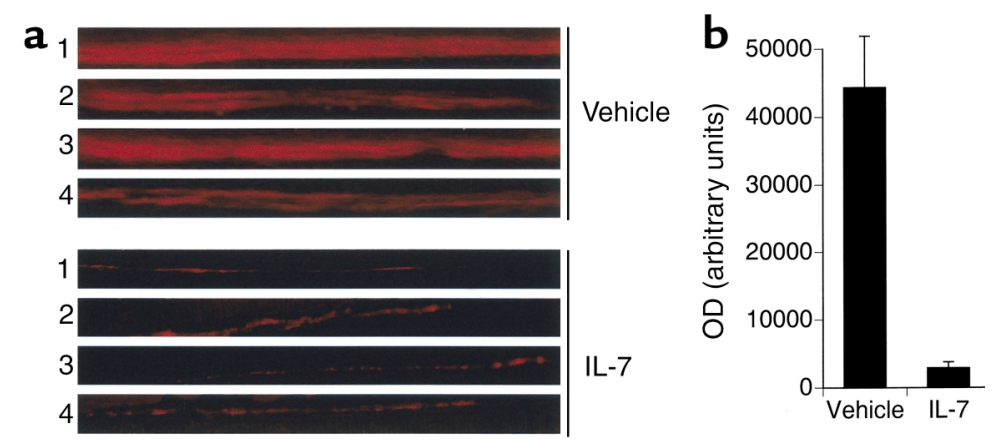

Figure 4

IL-7 inhibits bone formation in mice in vivo. Mice were injected intraperitoneally with IL-7 $(10 \mu \mathrm{g} / \mathrm{kg}$ body weight) or vehicle (PBS plus $0.1 \% \mathrm{BSA})$ for 30 days and pulsed with calcein 7 and 3 days prior to sacrifice. Tibiae were plastic-embedded, sectioned, and examined by fluorescence microscopy. (a) Representative calcein fluorescence bands from four different mice are shown for each group. Bands (1 $\mathrm{mm}$ in length) were photographed at comparable anatomic positions at the mid-diaphysis of each bone at $\times 100$ magnification. (b) Fluorescence intensity of the entire area of each of the four individual panels shown in $\mathbf{a}$, for both vehicleand IL-7-treated groups, was quantitated by densitometry using NIH Image. Data represent the average OD of four panels per group \pm SEM. 


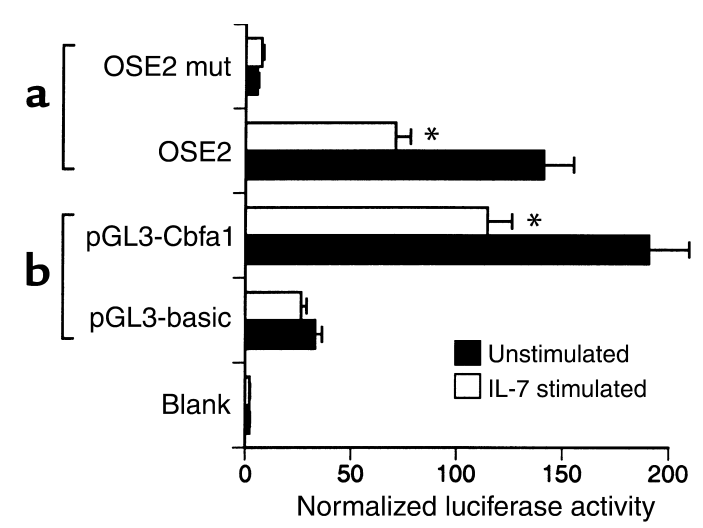

Figure 5

IL-7 suppresses the transactivation of a promoter driven by six OSE2 elements and blunts Cbfa1/Runx2 promoter activity in ROS 17/2.8 osteoblastic cells. (a) p6OSE2-luc, a luciferase reporter construct driven by six tandem copies of OSE2 fused to a minimal OCN promoter, and p6OSE2mut-luc, a control construct containing six inactive OSE2 sites, were transfected into ROS $17 / 2.8$ osteoblastic cells, and luciferase activity was assessed at 24 hours in unstimulated cells and cells stimulated with IL-7 $(10 \mathrm{ng} / \mathrm{ml})$. Data are shown as average \pm SD of triplicate cultures and are representative of three independent experiments. ${ }^{*} P<0.05$ ( $t$ test). (b) pGL3-Cbfa1/Runx2, a luciferase reporter construct containing the distal OB-specific Cbfa1/Runx2 promoter (-620 bp), or empty vector (pGL3-basic) was transfected into ROS $17 / 2.8$ osteoblastic cells, and luciferase activity was assessed at 24 hours in unstimulated cells and cells stimulated with IL-7 $(10 \mathrm{ng} / \mathrm{ml})$. The data are shown as average \pm SD of triplicate cultures and are representative of three independent experiments. ${ }^{*} P<0.05$ ( $t$ test $)$.

following IL-7 stimulation by examining the direct effect of IL-7 on the Cbfa1/Runx2 promoter. The distal OB-specific Cbfa1/Runx2 promoter (18) was cloned into a pGL3-basic luciferase expression vector (see Methods) and transfected into ROS 17/2.8 cells. Cells were stimulated with IL-7 $(10 \mathrm{ng} / \mathrm{ml})$ or with vehicle (PBS plus $0.1 \% \mathrm{BSA}$ ), and luciferase activity was measured by luminometer after 24 hours. Consistent with the previous data, IL-7 blunted the transactivation activity of the Cbfa1/Runx2 promoter in ROS 17/2.8 cells by $45 \%$ (Figure $5 \mathrm{~b}$ ). The downregulation of Cbfa1/Runx2 transcription in IL-7-stimulated cells is consistent with the hypothesis that IL-7 is a negative regulator of $\mathrm{OB}$ differentiation and activity.

IL-7 inhibits OPG production in osteoblastic cells. E2 has been shown to stimulate the RANKL decoy receptor OPG, a powerful inhibitor of osteoclastogenesis in osteoblastic

\section{Figure 6}

IL-7 inhibits OPG production in ROS $17 / 2.8$ cells. (a) Using semiquantitative RT-PCR, we measured OPG mRNA at 24 hours in ROS $17 / 2.8$ cells stimulated with vehicle (PBS plus $0.1 \%$ BSA) or IL-7 ( 10 $\mathrm{ng} / \mathrm{ml}$ ). Data are representative of two independent experiments. (b) OPG mRNA bands in a were quantitated by densitometry and normalized against GAPDH. (c) OPG protein production was measured by Western blot at 24 hours in primary calvarial OBs stimulated with vehicle (PBS plus $0.1 \%$ BSA) or IL-7 $(10 \mathrm{ng} / \mathrm{ml})$. Data are representative of two independent experiments. (d) OPG protein concentrations in $\mathbf{c}$ were quantitated by densitometry. cells $(29,30)$. Alterations in the relative ratio of RANKL to OPG in the bone marrow microenvironment have been suggested to be a major cause of bone loss in many metabolic disorders, including E2 deficiency (31). Recently it was reported that OPG is upregulated by Cbfa1/Runx2-responsive OSE2 sites in OBs (11). As our previous data demonstrate that IL-7 treatment of OBs leads to the downregulation of $\mathrm{Cbfa} 1 / \mathrm{Run} \times 2$ transcription, this suggests that IL-7 may also downregulate OPG production in OBs. To verify this prediction, we used semiquantitative RT-PCR to measure OPG mRNA in ROS 17/2.8 cells treated with vehicle (PBS plus $0.1 \%$ BSA) or with IL-7 for 24 hours (Figure 6a). The data show that IL-7 downregulates OPG mRNA production by $60 \%$ (Figure 6b). To verify these data on OPG protein levels in nontransformed murine OBs, OPG protein expression in primary calvarial OBs was evaluated by Western blot (Figure 6c). By densitometric quantitation, OPG was found to be decreased 50\% following IL-7 treatment (Figure 6d).

\section{Discussion}

The mechanism by which E2 deficiency drives uncoupling remains poorly elucidated. We now provide evidence that induction of IL-7 during E2 deficiency leads to a perturbation in bone homeostasis by targeting both bone formation and bone resorption. Based on our findings, we propose that IL-7 is one of the long-soughtafter cytokines that mediate uncoupling of bone formation from bone resorption during E2 deficiency.

We believe that this study provides the first demonstration that E2 withdrawal upregulates the levels of IL-7 in the bone marrow, and that IL-7 neutralization in vivo completely prevents bone loss following ovx. Although the mechanism by which ovx upregulates IL-7 remains to be determined, these observations demonstrate that IL-7 plays a central role in the pathogenesis of bone loss associated with E2 deficiency.

Surprisingly, we found that an additional mechanism by which IL-7 neutralization prevents ovx-induced bone loss is stimulation of bone formation, a finding which suggests that IL-7 is an antianabolic factor in bone.
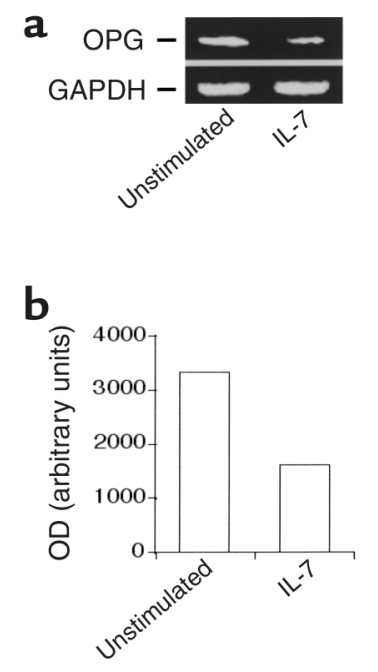
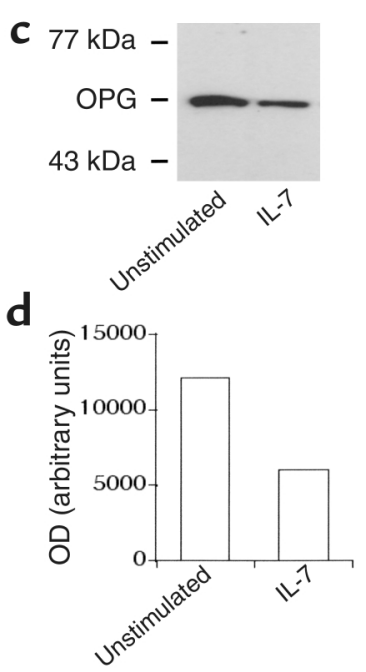
As IL-7 treatment of osteoblastic cells led to a decrease in the promoter activity of Cbfa1/Runx2 (Figure $5 \mathrm{~b}$ ), this suggests that, by decreasing Cbfa1/Runx2 transcription, IL-7 ultimately leads to a decrease in the protein concentration of Cbfa1/Runx2 and hence reduced transactivation of Cbfa1/Runx2-responsive promoters. This is evidenced by IL-7-induced repression of the Cbfa1/Runx2-driven promoter construct (p6OSE2-luc) and is consistent with the observed decrease in OPG mRNA and protein expression in response to IL-7, as well as with the enhanced levels of OCN protein detected in vivo following IL-7 neutralization. A $50 \%$ decrease in Cbfa1/Runx2 promoter activity correlated with a $45 \%$ decrease in the activity of the Cbfa1/Runx2-driven promoter construct p6OSE2-luc, and an approximately 50-60\% inhibition of OPG mRNA and protein production. These data suggest that the observed decrease in Cbfa1/Runx2 transcription induced by IL-7 directly accounts for the observed inhibitory effects of IL-7 on Cbfa1/Runx2driven genes. It should be pointed out, however, that Cbfa1/Runx2 regulation is extremely complex and that numerous additional mechanisms have been reported that could play an additional role in IL-7's regulation of $\mathrm{Cbfa} 1 / \mathrm{Runx} 2$. These mechanisms include modulation of Cbfa1/Runx 2 mRNA translation (32) and enhanced activity via phosphorylation by mitogen-activated protein kinases (MAPKs) (33). In addition, Cbfa1/Runx2 transactivation of $\mathrm{OCN}$ is regulated by means of direct protein-protein associations with other transcription factors, such as Msx2, a repressor of Cbfa1/Runx2 transcriptional activity (34). Msx2 is in turn repressed by protein-protein interactions with MINT (35) and Dlx5 (36).

Cbfa1/Runx2 is both a marker of and a key participant in the process of OB differentiation. Thus, whether IL-7 directly downregulates Cbfa1/Runx2 transcription, leading to a suppression of OB differentiation, or indirectly decreases Cbfa1/Runx2 transcription by inhibiting the differentiation of the OBs remains to be determined.

IL-7 is known to stimulate OC formation by inducing the secretion of osteoclastogenic cytokines by $\mathrm{T}$ cells (5). Therefore, the finding that treatment with anti-IL-7 antibody prevents bone loss by decreasing bone resorption was not unexpected. However, this study provides novel evidence that osteoclastogenesis may be further stimulated by IL-7 through downregulation of OPG, the decoy receptor of RANKL, due to a reduction of Cbfa1/Runx2 levels that is related to IL-7 action on the OB. OPG is thought to play a major role in the antiresorptive action of E2 on bone. E2 has been reported to increase OPG $\mathrm{mRNA}$ and protein levels in both normal human OBs (29) and osteoblastic cell lines (30), and administration of recombinant OPG is capable of blocking ovx-associated bone loss in rats (37). It should be noted that, in our study, the magnitude of both the ovx-induced increase in IL-7 and the repressive effect of IL-7 on Cbfa1/Runx2 levels is modest. This is not in conflict with the hypothesis that IL-7 is a relevant causal agent of the bone loss induced by E2 withdrawal, as the stimulatory effect of IL-7 on bone resorption is compounded by the inhibition of bone formation.

Our study supports the notion that E2 prevents bone loss by regulating the production of, and the responsiveness to, several inflammatory cytokines and hematopoietic growth factors. The novel finding that IL-7 is one such factor adds to the ongoing controversy regarding the relevance in vivo of the regulatory effects of each of the presently known E2-regulated factors. Such controversy stems from reports demonstrating that ovx-induced bone loss can be equally prevented by silencing any of several cytokines implicated in its pathogenesis. Among them are IL-1 $(38,39)$, IL-6 (40), TNF- $\alpha(12,39)$, and M-CSF (15). This apparent redundancy is likely to be explained by the fact that cytokines often regulate target cells in a synergistic manner. Furthermore, their production is under reciprocal control. As a result, a small repressive effect of $\mathrm{E} 2$ on one cytokine may be greatly amplified by another.

Another mechanism by which ovx causes bone loss is by increasing $\mathrm{T}$ cell TNF- $\alpha$ production, a phenomenon that is, in turn, caused by an increase in the number of T cells (12). Moreover, since TNF- $\alpha$ induces the stromal cell production of IL-7 (5), ovx further increases IL-7 production by upregulating $\mathrm{T}$ cell TNF- $\alpha$ production. The net result of this process is a significant increase in the $\mathrm{T}$ cell production of both RANKL and TNF- $\alpha$. Since these two cytokines stimulate OC formation in a synergistic manner $(12,41,42)$, upregulation of $\mathrm{T}$ cell number and of $\mathrm{T}$ cell effector function plays a key role in inducing the bone loss that follows ovx.

In summary, the findings described here suggest a model whereby upregulation of IL-7, a consequence of E2 deficiency, plays a central role in ovx-induced bone loss by uncoupling bone formation from bone resorption. IL-7 achieves this by upregulation of osteoclastogenesis, while simultaneously limiting the magnitude of the compensatory rise in bone formation necessary to compensate for enhanced bone resorption and to restore bone homeostasis.

\section{Acknowledgments}

The authors thank F. Patrick Ross for helpful advice, and Leonard Rifas for useful discussions and review of the manuscript. We thank Stewart D. Lyman for providing IL-7 antibody and hybridoma, Adrian Minty for recombinant human IL-7, Genetics Institute Inc. for recombinant human BMP2, and Gerard Karsenty for OSE2 constructs. M.N. Weitzmann was supported in part by grants from the National Institute of Arthritis and Musculoskeletal and Skin Diseases (AR-46375) and the Barnes-Jewish Hospital Research Foundation. R. Pacifici was supported in part by grants from the NIH (AR-41412 and AG-13534), the Eastern Missouri Chapter of the Arthritis Foundation, and the Lilly Center for Women's Health. 
1. Manolagas, S.C., and Jilka, R.L. 1995. Bone marrow, cytokines, and bone remodeling. N. Engl. J. Med. 332:305-311.

2. Miyaura, C., et al. 1997. Increased B-lymphopoiesis by interleukin 7 induces bone loss in mice with intact ovarian function: similarity to estrogen deficiency. Proc. Natl. Acad. Sci. USA. 94:9360-9365.

3. Hofmeister, R., et al. 1999. Interleukin-7: physiological roles and mechanisms of action. Cytokine Growth Factor Rev. 10:41-60.

4. Fry, T.J., and Mackall, C.L. 2002. Interleukin-7: from bench to clinic. Blood. 99:3892-3904.

5. Weitzmann, M.N., Cenci, S., Rifas, L., Brown, C., and Pacifici, R. 2000. Interleukin-7 stimulates osteoclast formation by up-regulating the T-cell production of soluble osteoclastogenic cytokines. Blood. 96:1873-1878

6. Sato, T., Shibata, T., Ikeda, K., and Watanabe, K. 2001. Generation of bone-resorbing osteoclasts from B220+ cells: its role in accelerated osteoclastogenesis due to estrogen deficiency. J. Bone Miner. Res. 16:2215-2221.

7. Karsenty, G. 1999. The genetic transformation of bone biology. Genes Dev. 13:3037-3051.

8. Kern, B., Shen, J., Starbuck, M., and Karsenty, G. 2001. Cbfa1 contributes to the osteoblast-specific expression of Type I collagen genes. J. Biol. Chem. 276:7101-7107.

9. Hoshi, K., Komori, T., and Ozawa, H. 1999. Morphological characterization of skeletal cells in Cbfa1-deficient mice. Bone. 25:639-651.

10. Schinke, T., and Karsenty, G. 1999. Characterization of Osf1, an osteoblast-specific transcription factor binding to a critical cis-acting element in the mouse Osteocalcin promoters. J. Biol. Chem. 274:30182-30189.

11. Thirunavukkarasu, K., et al. 2000. The osteoblast-specific transcription factor $\mathrm{Cbfa} 1$ contributes to the expression of osteoprotegerin, a potent inhibitor of osteoclast differentiation and function. J. Biol. Chem. 275:25163-25172.

12. Cenci, S., et al. 2000. Estrogen deficiency induces bone loss by enhancing T-cell production of TNF-alpha. J. Clin. Invest. 106:1229-1237.

13. Grabstein, K.H., et al. 1993. Inhibition of murine B and T lymphopoiesis in vivo by an anti-interleukin 7 monoclonal antibody. J. Exp. Med. 178:257-264.

14. Bhatia, S.K., Tygrett, L.T., Grabstein, K.H., and Waldschmidt, T.J. 1995. The effect of in vivo IL-7 deprivation on T cell maturation. J. Exp. Med. 181:1399-1409.

15. Cenci, S., Weitzmann, M.N., Gentile, M.A., Aisa, M.C., and Pacifici, R. 2000. M-CSF neutralization and egr-1 deficiency prevent ovariectomyinduced bone loss. J. Clin. Invest. 105:1279-1287.

16. Carpio, L.C., and Dziak, R. 1998. Activation of phospholipase D signaling pathway by epidermal growth factor in osteoblastic cells. J. Bone Miner. Res. 13:1707-1713.

17. Andrews, N.C., and Faller, D.V. 1991. A rapid micropreparation technique for extraction of DNA-binding proteins from limiting numbers of mammalian cells. Nucleic Acids Res. 19:2499.

18. Fujiwara, M., et al. 1999. Isolation and characterization of the distal promoter region of mouse Cbfa1. Biochim. Biophys. Acta. 1446:265-272.

19. Ducy, P., and Karsenty, G. 1995. Two distinct osteoblast-specific cis-acting elements control expression of a mouse osteocalcin gene. Mol. Cell. Biol. 15:1858-1869.

20. Traianedes, K., Dallas, M.R., Garrett, I.R., Mundy, G.R., and Bonewald, L.F. 1998. 5-Lipoxygenase metabolites inhibit bone formation in vitro. Endocrinology. 139:3178-3184.

21. Delmas, P.D., Schlemmer, A., Gineyts, E., Riis, B., and Christiansen, C. 1991. Urinary excretion of pyridinoline crosslinks correlates with bone turnover measured on iliac crest biopsy in patients with vertebral osteoporosis. J. Bone Miner. Res. 6:639-644.
22. Brown, J.P., et al. 1984. Serum bone Gla-protein: a specific marker for bone formation in postmenopausal osteoporosis. Lancet. 1:1091-1093.

23. Lee, M.H., et al. 1999. Transient upregulation of CBFA1 in response to bone morphogenetic protein- 2 and transforming growth factor beta1 in C2C12 myogenic cells coincides with suppression of the myogenic phenotype but is not sufficient for osteoblast differentiation. J. Cell. Biochem. 73:114-125.

24. Hale, L.V., Ma, Y.F., and Santerre, R.F. 2000. Semi-quantitative fluorescence analysis of calcein binding as a measurement of in vitro mineralization. Calcif. Tissue Int. 67:80-84.

25. Karsenty, G., et al. 1999. Cbfa 1 as a regulator of osteoblast differentiation and function. Bone. 25:107-108.

26. Ducy, P., et al. 1999. A Cbfa1-dependent genetic pathway controls bone formation beyond embryonic development. Genes Dev. 13:1025-1036.

27. Ducy, P., Zhang, R., Geoffroy, V., Ridall, A.L., and Karsenty, G. 1997. Osf2/Cbfa1: a transcriptional activator of osteoblast differentiation. Cell. 89:747-754.

28. Drissi, H., et al. 2000. Transcriptional autoregulation of the bone related CBFA1/RUNX2 gene. J. Cell. Physiol. 184:341-350.

29. Hofbauer, L.C., et al. 1999. Estrogen stimulates gene expression and protein production of osteoprotegerin in human osteoblastic cells Endocrinology. 140:4367-4370.

30. Saika, M., Inoue, D., Kido, S., and Matsumoto, T. 2001. 17beta-Estradiol stimulates expression of osteoprotegerin by a mouse stromal cell line, ST-2, via estrogen receptor-alpha. Endocrinology. 142:2205-2212.

31. Hofbauer, L.C., et al. 2000. The roles of osteoprotegerin and osteoprotegerin ligand in the paracrine regulation of bone resorption. J. Bone Miner. Res. 15:2-12.

32. Sudhakar, S., Li, Y., Katz, M.S., and Elango, N. 2001. Translational regulation is a control point in RUNX2/Cbfa1 gene expression. Biochem. Biophys. Res. Commun. 289:616-622.

33. Xiao, G., et al. 2000. MAPK pathways activate and phosphorylate the osteoblast-specific transcription factor, Cbfa1. J. Biol. Chem. 275:4453-4459.

34. Shirakabe, K., Terasawa, K., Miyama, K., Shibuya, H., and Nishida, E. 2001. Regulation of the activity of the transcription factor Runx 2 by two homeobox proteins, Msx2 and Dlx5. Genes Cells. 6:851-856.

35. Newberry, E.P., Latifi, T., and Towler, D.A. 1999. The RRM domain of MINT, a novel Msx2 binding protein, recognizes and regulates the rat osteocalcin promoter. Biochemistry. 38:10678-10690.

36. Newberry, E.P., Latifi, T., and Towler, D.A. 1998. Reciprocal regulation of osteocalcin transcription by the homeodomain proteins Msx2 and Dlx5. Biochemistry. 37:16360-16368.

37. Simonet, W.S., et al. 1997. Osteoprotegerin: a novel secreted protein involved in the regulation of bone density. Cell. 89:309-319.

38. Kimble, R.B., et al. 1994. Interleukin-1 receptor antagonist decreases bone loss and bone resorption in ovariectomized rats. J. Clin. Invest. 93:1959-1967.

39. Kimble, R.B., et al. 1995. Simultaneous block of interleukin-1 and tumor necrosis factor is required to completely prevent bone loss in the early postovariectomy period. Endocrinology. 136:3054-3061.

40. Jilka, R.L., et al. 1992. Increased osteoclast development after estrogen loss: mediation by interleukin-6. Science. 257:88-91.

41. Zhang, Y.H., Heulsmann, A., Tondravi, M.M., Mukherjee, A., and AbuAmer, Y. 2001. Tumor necrosis factor-alpha (TNF) stimulates RANKLinduced osteoclastogenesis via coupling of TNF type 1 receptor and RANK signaling pathways. J. Biol. Chem. 276:563-568.

42. Lam, J., et al. 2000. TNF-alpha induces osteoclastogenesis by direct stimulation of macrophages exposed to permissive levels of RANK ligand. J. Clin. Invest. 106:1481-1488. 